\title{
Metastatic pulmonary calcification diagnosed by transbronchial cryobiopsy
}

\author{
Daniel Samolski ${ }^{1}$, Roberto Duré1, Gonzalo Bentolila² ${ }^{2}$, Teresa Castiglioni ${ }^{3}$ \\ ${ }^{1}$ Bronchoscopy Unit, Francisco Muñiz Hospital, Buenos Aires; ${ }^{2}$ Fundaleu, Buenos Aires; ${ }^{3}$ Elsner Pathology Center, \\ Buenos Aires, Argentina
}

\begin{abstract}
Metastatic pulmonary calcification is a rare disease characterized by calcium deposits in the lung. We describe a case where this pathology is observed associated with bone lithic lesions and kidney failure and it was diagnosed with transbronchial cryobiopsy.
\end{abstract}

\section{Introduction}

Metastatic pulmonary calcification (MPC) is a disease characterized by calcium deposits in a previously healthy lung, which differentiates it from the dystrophic calcification which affects a

Correspondence: Daniel Samolski, Bronchoscopy Unit, Francisco Muñiz Hospital, 2272 Uspallata st, Buenos Aires C1282AEN, Argentina.

Tel. +54.11.4305-8537.

E-mail: dsamolski@gmail.com

Key words: Interstitial lung disease; metastatic pulmonary calcification; transbronchial cryobiopsy.

Conflict of interest: The authors declare that they have no conflict of interest.

Contributions: DS, RD, participated actively in the diagnostic procedure of the patient and wrote the main part of the manuscript; GB, hematological diagnosis and carried out treatment; TC, carried out the pathologic study and reviewed the manuscript. All the authors have read and approved the final manuscript and agree to be accountable for all aspects of the work in ensuring that questions related to the accuracy or integrity of any part of the work are appropriately investigated and resolved

Ethical approval: Ethical approval was not acquired since this manuscript is a case report and was not produced in the context of a research trial. Informed consent acquired during hospitalization and bronchoscopy was considered sufficient.

Received for publication: 4 June 2020.

Accepted for publication: 8 July 2020.

${ }^{\circ}$ Copyright: the Author(s), 2020

Licensee PAGEPress, Italy

Monaldi Archives for Chest Disease 2020; 90:1430

doi: 10.4081/monaldi.2020.1430

This article is distributed under the terms of the Creative Commons Attribution Noncommercial License (by-nc 4.0) which permits any noncommercial use, distribution, and reproduction in any medium, provided the original author(s) and source are credited. previously damaged tissue. This entity is associated with benign or malignant diseases that present with severe hypercalcemia. Among the former the most common is chronic renal failure. Within the second, osteolytic metastasis [1-3].

\section{Case Report}

We present a male patient, 42 years old, with a medical history of hypothyroidism under replacement treatment with levothyroxine. He started on July 2019 with low back and rib pain associated with night sweats and weight loss. On August 2019, a CT scan was performed and lytic lesions in ribs, vertebrae, and pelvis were observed. For these findings, he was admitted to the hospital for study. In the laboratory it had high urea and creatinine levels (maximum values: urea $143 \mathrm{mg} / \mathrm{dl}$ and creatinine $3.9 \mathrm{mg} / \mathrm{dl}-$ BUN $66.7 \mathrm{mg} / \mathrm{dl}$ ) although not requiring dialytic treatment. The patient also had hypercalcemia with maximum blood total calcium $15.3 \mathrm{mg} / \mathrm{dl}(3.82 \mathrm{mmol} / \mathrm{L})$ and ionic calcium $1.84 \mathrm{mg} / \mathrm{dl}(0.46$ $\mathrm{mmol} / \mathrm{L}$ ), parathormone $18.3 \mathrm{pg} / \mathrm{ml}$ and bone alkaline phosphatase $>120 \mathrm{IU} / \mathrm{L}$ that were assumed secondary to tumor bone lysis and probable hyperparathyroidism secondary to impaired renal function. A kidney biopsy was performed showing acute tubulointerstitial nephritis, which could be related to the use of analgesics or, until then, an unrecognized systemic disease. High dose corticosteroids and pamidronate were administered. Although we initially thought about the diagnosis of multiple myeloma, the performance of an iliac crest biopsy diagnosed a diffuse B-cell non-Hodgkin lymphoma. He received chemotherapeutic treatment with $\mathrm{R}-$ $\mathrm{CHOP}$ scheme with good tolerance and reaching complete remission according to last PET CT.

Six months after his first hospitalization, the patient was referred to our Pulmonology Unit because of abnormalities at the chest CT scan in absolute absence of respiratory symptoms. It should be noted that in the first tomography performed at the hospital, the lung did not show any abnormality. The alterations appeared in a second image obtained already under chemotherapy treatment, one month after that hospitalization. The image (Figure 1) was characterized by bilateral and predominantly centrilobular ground glass opacities, affecting mainly upper and medium lobes and respecting the lower lobes almost entirely. Spirometry showed values in reference range including DLCO. Initially we thought about an infectious process or pulmonary toxicity due to drugs. Being a diffuse lung disease, we decided to perform a bronchoscopy with bronchoalveolar lavage (BAL) and transbronchial cryobiopsies $[4,5]$ to obtain a better diagnostic result. The BAL and four cryobiopsies were obtained from the middle lobe $\left(1 \mathrm{~cm}^{3}\right.$ as a whole). A moderate bleeding with the last biopsy prevented us from taking more material. The BAL showed no bacteria on direct examination neither in the culture. The stainings for acid fast 
bacilli and fungi were negative. Cytological BAL analysis showed $78 \%$ of alveolar macrophages, $1 \%$ of neutrophils leucocytes, $7 \%$ of lymphocytes and $14 \%$ of bronchial and paving cells. The histopathological report informed fragments of alveolar parenchyma with interstitial widening due to calcium deposits in the form of crystals or lumps, surrounded by numerous giant multinucleate foreign body cells. Associated interstitial fibrosis coexists, which stands out with Masson's trichrome. In the periphery there are numerous sectors of undamaged parenchyma. The alveolar cells are focally hyperplastic. Congo Red and thioflavin staining for amyloid deposits were negative. The morphological picture, in the clinical context of the patient, represents the entity "metastatic pulmonary calcification" (Figure 2).

\section{Discussion}

This entity represents a rare pathology that should be considered within the differential diagnoses of subacute and chronic lung diseases. Differential diagnoses both clinical and radiological include varied pathologies: infections, metastases of solid tumors, occupational and deposit diseases, among others. MPC is the most common cause of multifocal lung lesions in patients with chronic renal impairment and hypercalcemia and should be considered in the pres- ence of radiological alterations predominantly in upper lobes, a situation generated by the higher $\mathrm{paCO}_{2}$ (higher alkalinity) of the pulmonary apex that favor the deposit of calcium salts [1]. While the pathology is histologically interstitial, the tomographic image simulates a pathology of airspace. It is also common for calcifications to occur in other thoracic structures: chest wall vessels, myocardium, bronchial wall, pulmonary arteries, superior vena cava $[1,2]$. The image is not typically calcium in the parenchyma, being able to be explained by the small size of the calcium deposits and by technical issues related to the tomographic image (high kilovoltage).

Regarding the diagnostic technique we used transbronchial cryobiopsy according to the advantage of this technique in diffuse lung pathologies and the usefulness of this and associated bronchoalveolar lavage to rule out other pathologies considered. Most patients do not require any treatment if their involvement is asymptomatic and not progressive. In symptomatic patients the use of bisphosphonates and phosphorus chelating has been considered. Parathyroidectomy may even be reported in non-responder patients. Dialytic treatment is indicated in patients presenting this entity associated with advanced stage nephropathy [3]. The described patient remains asymptomatic four months after the pneumological diagnosis. It is free of oncohematological disease and has stabilized its renal function (creatinine $1.6 \mathrm{mg} / \mathrm{dl}$ - clearance of creatinine $65 \mathrm{ml} / \mathrm{min}$ ). Regarding the lung only clinicalradiological, evolutionary control has been indicated.
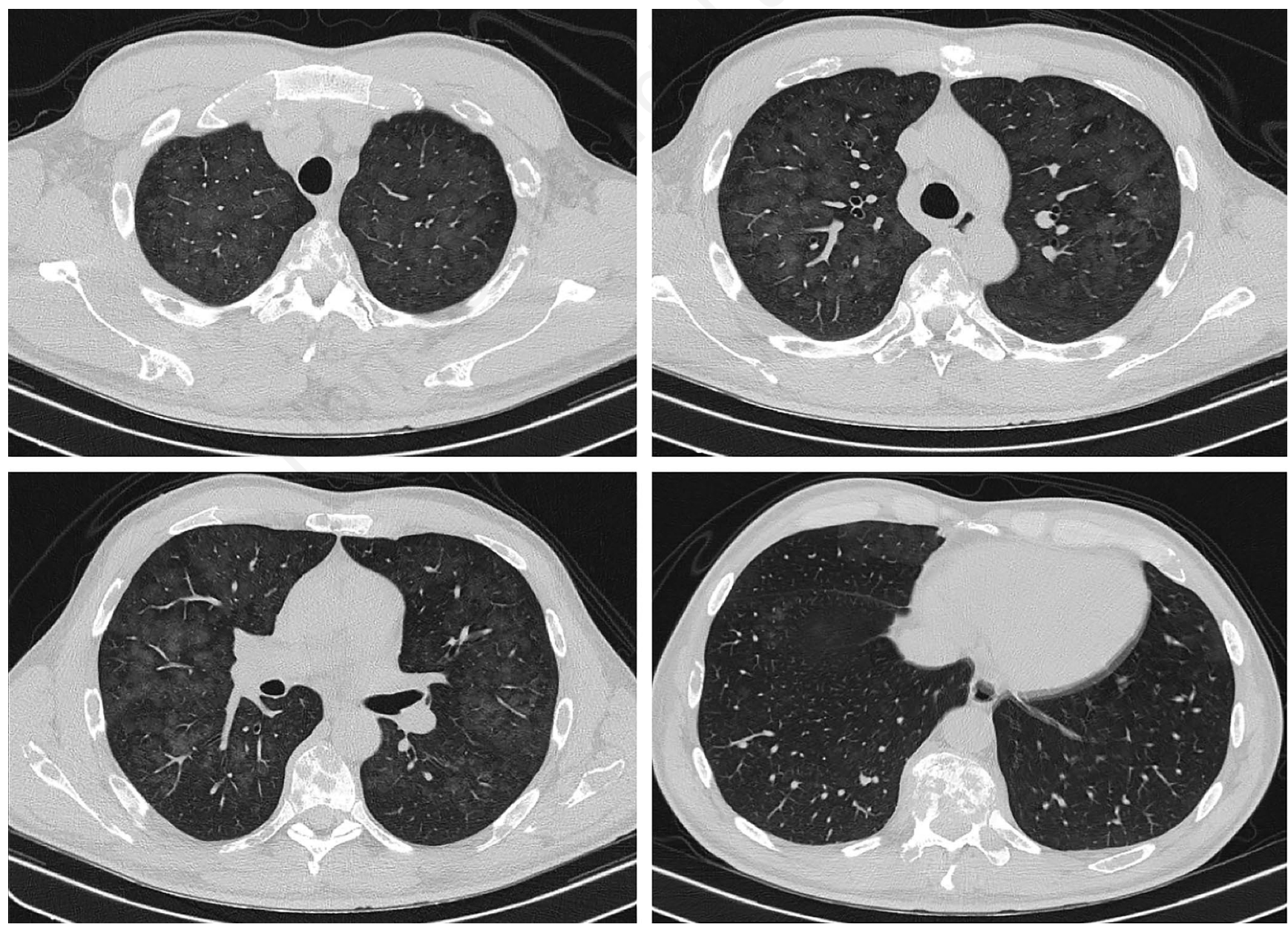

Figure 1. Chest CT scan showing bilateral diffuse alveolar involvement, centrilobular in appearance, with inferior lobe indemnity. 

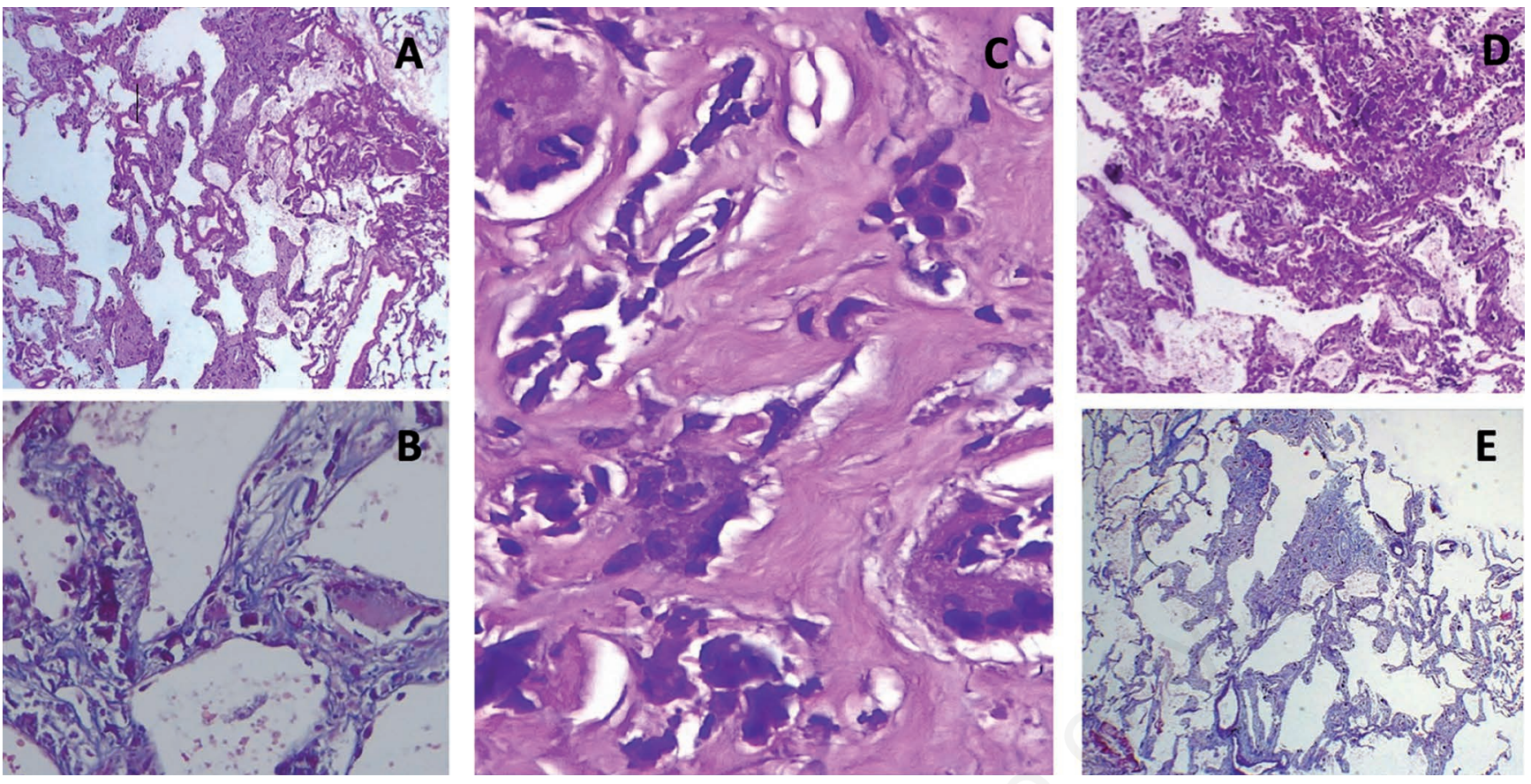

Figure 2. Transbronchial cryobiopsy. A) Thickening of alveolar walls due to fibrosis and calcium deposits; hematoxylin \& eosin (H\&E), 100x. B) Fibrosis of alveoli surrounding multinucleated histiocytes and calcium deposits; Masson's trichrome (MT), 400x. C) Gigantocellular reaction in the interstitium by calcium deposits (H\&E, 1000x). D) Lung with calcium deposits (H\&E; 400x). E) Thickening of alveolar walls due to fibrosis and calcium deposits (MT, 100x).

\section{Conclusions}

Pulmonary metastatic calcification is a rare benign entity that should be considered as a differential diagnosis in patients with hypercalcemia and pulmonary infiltrates. The diagnosis of metastatic pulmonary calcification by conventional transbronchial biopsy [6] has been described, but we have not found in the medical literature the use of cryobiopsy as a diagnostic method in this pathology. Transbronchial cryobiopsy [5] is shown as a minimally invasive and useful way to diagnose it.

\section{References}

1. Belem LC, Zanetti G, Soares Souza Jr A et al. Metastatic pul- monary calcification: State of the art review focuses on imaging findings. Resp Med J 2014;108:668-76.

2. Pasquier M, Schallet MD, Abdou M, Eckert P. [Les calcifications pulmonaires metastatiques].[Article in French]. Rev Mal Respir 2012;29:775-84.

3. Puy MC, Rodriguez Arias JM, Casan P. [Calcificaciones pulmonares asociadas a insuficiencia renal crónica].[Article in Spanish]. Arch Bronconeumol 2007;43:349-51.

4. Hetzel J, Maldonado F, Ravaglia C et al. Transbronchial cryobiopsies for the diagnosis of diffuse parenchymal lung disease: Expert statement from the cryobiopsy working group on safety and utility and a call for standardization of the procedure. Respiration 2018;95:188-200.

5. Pajares V, Torrego A, Puzo C et al. Utilización de criosondas para la realización de la biopsia pulmonar transbronquial. Arch Bronconeumol 2010;46:111-5.

6. Taniwaki M, Kawamoto K, Yamasaki M, et al. Severe pulmonary metastatic calcification. Am J Med 2019;132:e733-4. 